\title{
Necessidade de cuidado e funcionalidade entre idosas religiosas
}

The need for care and functionality among religious older women

Necesidad de cuidado y funcionalidad entre ancianas religiosas

Neuza Maria Sangiorgio Mozer ${ }^{1}$, Adriano Pasqualotti ${ }^{2}$, Marilene Rodrigues Portella ${ }^{3}$

${ }_{1}^{1}$ Fisioterapeuta, Mestre em Envelhecimento Humano. Passo Fundo, RS, Brasil. E-mail: ir.neuza@yahoo.com.br.

2 Matématico, Doutor em Informática na Educação. Professor Titular da da Universidade de Passo Fundo (UPF). Passo Fundo, RS, Brasil. E-mail: pasqualotti@upf.br.

${ }^{3}$ Enfermeira, Doutora em Enfermagem. Professora Titular da UPF. Passo Fundo, RS, Brasil. E-mail: portella@upf.br.

\section{RESUMO}

O objetivo foi avaliar a necessidade de cuidado autorreferida e a funcionalidade entre idosas religiosas. Trata-se de estudo transversal de cunho populacional. A coleta realizou-se com questionário estruturado contendo dados sociodemográficos, autorreferência para necessidade de cuidados e avaliação da funcionalidade, por meio do Index de Katz, averiguando independência nas atividades da vida diária (AVD). Os dados foram analisados por meio dos testes de Mann-Whitney e qui-quadrado, para um nível de significância de $p \leq 0,05$ e intervalo de confiança (IC) de 95\%. Apresentam resultados significativamente estatísticos para as variáveis banhar-se, vestir-se e ir ao banheiro. Concluise que, embora referindo necessidade de cuidado, as religiosas reservam a si, no máximo, o autocuidado. As evidências remetem à necessidade de ampliar os estudos sobre envelhecimento, congregando cenários similares, haja vista ser este processo progressivo e expressivo nas próprias congregações religiosas.

Descritores: Envelhecimento; Saúde da Mulher; Institucionalização; Avaliação.

\section{ABSTRACT}

The objective was to evaluate self-reported need for care and functionality among religious older women. It is a crosssectional population-based survey. Data collection took place via a structured questionnaire containing sociodemographic information, self-reported need for care and assessment of functionality, through the Katz Index, which investigated independence in activities of daily living (ADL). The data were analyzed using Mann-Whitney $U$ and chi-squared test, with a significance level of $p \leq 0.05$ and a $95 \%$ confidence interval (CI). The following variables presented statistical significance: bathing, dressing and toileting. We conclude that although they reported the need for care, at most, our participants provide themselves with self-care. Evidence points to the need for more widespread studies on ageing in similar scenarios, for this process is progressive and expressive in religious congregations.

Descriptors: Aging; Women's Health; Institutionalization; Evaluation.

\section{RESUMEN}

Se objetivó evaluar la necesidad de cuidado autorreferida y la funcionalidad entre ancianas religiosas. Estudio transversal de cuño poblacional. Recolección realizada con cuestionario estructurado incluyendo datos sociodemográficos, autorreferencia de necesidad de cuidados y evaluación de funcionalidad mediante Índice de Katz, averiguando independencia en actividades de la vida diaria (AVD). Los datos fueron analizados mediante los tests de Mann-Whitney y Chi-cuadrado, para un nivel de significatividad de $p \leq 0,05$ e intervalo de confianza (IC) de 95\%. Presentaron resultados significativamente estadísticos para las variables bañarse, vestirse e ir al baño. Se concluye en que aún refiriendo necesidad de cuidado, las religiosas reservan para sí al máximo el autocuidado. Las evidencias remiten a la necesidad de ampliar los estudios sobre envejecimiento, congregando escenarios similares, teniendo en cuenta que este proceso es progresivo y expresivo en las propias congregaciones religiosas.

Descriptores: Envejecimiento; Salud de la Mujer; Institucionalización; Evaluación. 


\section{INTRODUÇÃO}

O envelhecimento populacional acarreta aumento na quantidade de idosos dependentes, seja por consequência de uma doença crônica não transmissível ou pelo ônus do próprio envelhecimento ${ }^{(1)}$. Os avanços no padrão de vida, na disponibilidade de alimentos e nos recursos médicos e sanitários ocorridos durante o século $X X$ influenciaram na queda da taxa de mortalidade e na esperança de vida média da humanidade para ambos os sexos, porém os mais expressivos acréscimos foram observados na população feminina(2).

As mudanças fisiológicas inerentes ao envelhecimento quase sempre resultam em diminuição da capacidade funcional a médio e longo prazo, sendo que estas tornam os idosos mais suscetíveis à fragilidade e à dependência de cuidados ${ }^{(3)}$. A capacidade funcional é compreendida como a aptidão que a pessoa tem para realizar, de forma independente, as atividades da vida diária (AVD) consideradas essenciais para o autocuidado (4).

A longevidade quase sempre traz consigo eventos ou fatores que levam o indivíduo ao declínio de suas capacidades funcionais chegando até à necessidade de cuidados de longa duração (CLD). Historicamente, a atividade de cuidar, seja das crianças ou das gerações mais velhas, tem sido predominantemente feminina. No cuidado dos idosos dependentes as mulheres são as principais cuidadoras, como membros da família exercem sua atividade gratuitamente, rara vezes, nesta condição recebem auxílio financeiro(5). A maior sobrevida e longevidade feminina é uma realidade confirmada nos diversos países ${ }^{(2)}$. Para as mulheres brasileiras a esperança de vida ao nascer chega aos 77,32 anos(6), o que supõe um expressivo contingente feminino ocupando-se com o cuidado dos mais velhos.

Os fatores intrínsecos e extrínsecos que permeiam a vida dos indivíduos podem produzir consequências na velhice quase sempre associadas à dependência parcial ou total para a realização das atividades de autocuidado e possuem repercussões mais profundas do que simplesmente depender de um cuidador. O pudor que a maioria dos idosos preserva quanto à exposição do corpo, mesmo que para pessoas de sua intimidade, cria constrangimentos que podem levar à complicação de sua saúde ${ }^{(7)}$.

Por outro lado, a prevenção da instalação de processos incapacitantes é sugerida e estimulada de várias formas, incluindo prática de atividade física, exercícios de reabilitação funcional, hábitos de vida diária saudáveis entre outros, os quais são fundamentais para a manutenção da funcionalidade no processo de envelhecimento. Na literatura encontramos importantes trabalhos que vêm apontando a relação benéfica da prática de atividade física atuando na manutenção da capacidade funcional ${ }^{(8-9)}$.

O estudo abordou o cenário do envelhecimento na Vida Religiosa Consagrada (VRC) feminina diante da necessidade de cuidados de longa duração. Como vivem as religiosas idosas que necessitam de CLD? Quem são elas? Quais suas condições de saúde e funcionalidade para as AVD? Qual a tipologia de cuidados que aportam?

No Brasil tem se observado que a temática acerca dos idosos institucionalizados está em evidência na produção científica, porém, o envelhecimento das congregações religiosas não segue a tendência dos estudos gerontológicos. Assim, acreditamos que o presente artigo venha contribuir com novos conhecimentos nessa área a partir do olhar lançado às comunidades de idosas religiosas. Depositamos confiança na obtenção de subsídios, os quais possam agregar informações para outras congêneres que exercitam CLD nesse contexto. O objetivo foi avaliar fatores associados à necessidade de cuidado autorreferida e à funcionalidade entre idosas religiosas.

\section{MÉTODOS}

Estudo transversal de cunho populacional e foi desenvolvido em uma cidade da região norte do Rio Grande do Sul - Brasil em residências destinadas aos CLD dos membros idosos pertencentes a três congregações religiosas, envolvendo mulheres acima de 60 anos.

A amostra foi constituída por 50 idosas religiosas e o critério considerado para fazer parte do mesmo foi ter 60 anos ou mais e estar morando nas residências destinadas aos CLD das congregações às quais são pertinentes independentemente da condição de cuidadoras ou necessitadas de cuidados.

A coleta realizou-se por meio de entrevista no período de outubro de 2011 a janeiro de 2012 empregando um questionário estruturado contendo entre outros, dados sociodemográficos, autorreferência para necessidade de cuidados e avaliação da funcionalidade, obtida com o Index de Katz para investigar a independência nas AVD. Criado por Sidney Katz e publicado pela primeira vez em 1963, até hoje é um dos instrumentos mais usados em estudos com idosos, devido à praticidade na aplicação e confiabilidade, o que respalda a escolha, aliado ao fato do mesmo ser recomendado pelo Ministério da Saúde ${ }^{(10)}$. As variáveis 
contempladas para avaliação funcional são descritas como AVD e relacionadas ao autocuidado, como banharse, vestir-se, ir ao banheiro, transferir-se, continência e alimentar-se, classificando os indivíduos como independentes, parcialmente dependentes ou dependentes $^{(11)}$.

Os dados foram analisados utilizando o SPSS 18.0. Para avaliar a associação entre as variáveis aplicaram-se os testes de Mann-Whitney e qui-quadrado. Considerouse um nível de significância de $p \leq 0,05$ e intervalo de confiança (IC) de $95 \%$. Os indivíduos foram classificados como independentes e com dependência nas variáveis relacionadas às necessidades de cuidado autorreferida.

A realização do estudo foi autorizada pelas Coordenadoras das respectivas Residências, observando os princípios éticos as participantes assinaram o Termo de Consentimento Livre e Esclarecido e o Comitê de Ética e Pesquisa da Universidade de Passo Fundo aprovou a investigação, protocolo n. 312/2011.

\section{RESULTADOS}

A população de pesquisa compreendia um total de 54 religiosas idosas. Participaram do estudo 50 idosas. A média de idade foi de 82,3 anos, com um desvio padrão (DP) de 9,3 anos. Quanto ao tempo de vida religiosa, a média foi de 60,6, com um desvio padrão de 9,9. Relativo à escolaridade, a média de anos de estudo ficou em 11,3 anos, com um desvio padrão de 4,6 anos. No que confere a atenção à pessoa idosa, a maioria, 38 (76,0\%) acusou necessidade de cuidado. Por outro lado, oito $(16,0 \%)$ passaram a ser cuidadoras ao serem transferidas para as residências de CLD. Referente à escolaridade, a média de anos de estudo ficou em 11,3 anos, com um desvio padrão de 4,6 anos.

A Tabela 1 apresenta a relação entre as necessidades de cuidado versus idade, tempo de vida religiosa e anos de estudos.

Tabela 1: Necessidades de cuidado versus idade, tempo de vida religiosa e anos de estudos. Passo Fundo, RS, Brasil, 2012.

\begin{tabular}{ccccc}
\hline Categorias & Necessidades de cuidado & Média & Desvio padrão & p \\
\hline \multirow{2}{*}{ Idade } & Sim & 85,1 & 8,0 & $<0,000^{*}$ \\
& Não & 73,7 & 8,1 & \multirow{2}{*}{$0,004^{*}$} \\
\multirow{2}{*}{ Tempo de vida religiosa } & Sim & 62,6 & 9,5 & \\
& Não & 54,3 & 8,9 & $0,001^{*}$ \\
\hline \multirow{2}{*}{ Anos de estudos } & Sim & 10,1 & 4,0 & 0,1 \\
\hline
\end{tabular}

Teste de Mann-Whitney; * valor significativo para um $\mathrm{p} \leq 0,05$.

A necessidade de cuidados autorreferida foi analisada dicotomicamente com três variáveis: idade, tempo de vida religiosa e anos de estudo. Quando relacionada à idade os indivíduos que afirmaram necessidade de cuidado apresentam média de 85,1 anos, com um desvio padrão de 8,0 anos. Quanto ao tempo de vida religiosa, a prevalência de necessidade de cuidados foi manifestada entre os que apresentam mais tempo na instituição, com média de 62,6 anos, com um desvio padrão de 9,5 anos. Quanto à relação entre anos de estudo, a necessidade de cuidado foi manifesta entre os indivíduos que apresentam menor média de anos de estudo 10,1 anos, com um desvio padrão de 4,0 anos. Destaca-se que todas as variáveis foram estatisticamente significativas quando relacionadas às necessidades de cuidado

A Tabela 2 apresenta a relação entre a capacidade funcional para as AVD segundo as variáveis do Index de Katz e a necessidade de cuidado autorreferida para a realização das AVD.

Destacam-se valores estatisticamente significativos para a necessidade de cuidado autorreferida quando relacionada às variáveis: banhar-se $(p=0,006)$, vestirse $(p=0,004)$, ir ao banheiro a tempo $(p=0,006)$ e transferência $(0,045)$.

Quanto à prática de exercício físico, 26 (52, 0\%) praticam raramente, 20 (40,0\%) praticam com frequência e três $(6,0 \%)$ nunca realizavam atividade física e uma parou de praticar atividade física. A funcionalidade foi analisada no desempenho de seis funções para as AVD.

A funcionalidade, avaliada através do index de Katz, analisou o desempenho de seis funções para as AVD. Quanto a alimentar-se, $35 \quad(70,0 \%)$ relataram independência para a tarefa. Já no item transferência, 28 $(56,0 \%)$ consegue fazê-lo sem dificuldade, sendo a mesma prevalência para a continência. Vestir-se, 25 $(50,0 \%)$ realizam a tarefa sem dificuldade. Para banharse, $23(46,0 \%)$ conseguem fazê-lo de forma independente. 
Tabela 2: Fatores associados à necessidade de cuidado autorreferida e funcionalidade. Passo Fundo, RS, Brasil, 2012.

\begin{tabular}{|c|c|c|c|c|c|}
\hline \multirow{2}{*}{ Variáveis } & \multirow{2}{*}{ Categorias } & \multirow{2}{*}{ Total } & \multicolumn{2}{|c|}{ Necessidade de cuidado autorreferida } & \multirow{2}{*}{$\mathbf{p}$} \\
\hline & & & Sim & Não & \\
\hline \multirow{2}{*}{ Banhar } & Independente & 23 & $13(56,5)$ & $10(43,5)$ & \multirow{2}{*}{0,006} \\
\hline & Com dependência & 27 & $25(92,6)$ & $2(7,4)$ & \\
\hline \multirow{2}{*}{ Vestir } & Independente & 25 & $14(56,0)$ & $11(44,0)$ & \multirow{2}{*}{0,002} \\
\hline & Com dependência & 25 & $24(96,0)$ & $1(4,0)$ & \\
\hline \multirow{2}{*}{ Ir ao banheiro a tempo } & Independente & 28 & $17(60,7)$ & $11(39,3)$ & \multirow{2}{*}{0,006} \\
\hline & Com dependência & 22 & $21(95,5)$ & $1(4,5)$ & \\
\hline \multirow{2}{*}{ Transferência } & Independente & 28 & $18(64,3)$ & $10(35,7)$ & \multirow{2}{*}{0,045} \\
\hline & Com dependência & 22 & $20(90,9)$ & $2(9,1)$ & \\
\hline \multirow{2}{*}{ Continência } & Independente & 28 & $19(67,9)$ & $9(32,1)$ & \multirow{2}{*}{0,186} \\
\hline & Com dependência & 22 & $19(86,4)$ & $3(13,6)$ & \\
\hline \multirow{2}{*}{ Alimentar-se } & Independente & 35 & $24(68,8)$ & $11(31,2)$ & \multirow{2}{*}{0,079} \\
\hline & Com dependência & 15 & $14(93,3)$ & $1(6,7)$ & \\
\hline
\end{tabular}

Com dependência: Parcialmente dependente/dependente; Teste de qui-quadrado; * valor significativo para um $p \leq 0,05$.

\section{DISCUSSÃo}

A dependência e a incapacidade funcional observadas nos resultados do estudo em questão não podem negligenciar o fato de a amostra apresentar alta média de idade, concordando com os resultados de investigações que apontam que a idade de 80 anos ou mais acarreta maior propensão à dependência e no avanço da idade, aumentam as chances de ocorrências desfavoráveis na capacidade funcional ${ }^{(8,12-13,)}$.

Atividades relacionadas ao autocuidado, como banhar-se, vestir-se e ir ao banheiro demonstraram-se comprometidas para um número significativo dos indivíduos avaliados da pesquisa. Estudos realizados com idosos concordam que na evolução da faixa etária ocorre maior nível de dependência para AVD e que capacidade funcional inadequada, associa-se a idade maior ou igual a 70 anos e ser do sexo feminino ${ }^{(10,14)}$.

Ressalta-se a alta média de anos de estudos encontrada entre os indivíduos e sua relação com a necessidade de cuidado, dado que se contrapõem às pesquisas que encontraram na baixa escolaridade um dos fatores mais fortemente associados à incapacidade funcional em idosas brasileiras ${ }^{(15-16)}$.

$\mathrm{Na}$ avaliação da atividade ir ao banheiro, os dados da pesquisa corroboram com resultados apontados por outros estudos realizados com idosos os quais sinalizaram a dependência para esta AVD, associado a necessidade de cuidado em um número significativo de indivíduos ${ }^{(11,17-21)}$.

Foi encontrada também diferença significativa para a atividade de vestir-se, discordando de outro estudo no qual não foram observados resultados significativos quando se relacionou a idade com o desempenho de idosos na realização das atividades propostas pelo Índice de Katz, inclusive para esta atividade ${ }^{(18)}$.

As idosas apresentaram maior dependência funcional para as atividades tomar banho e vestir-se, corroborando com resultados encontrados em outros estudos que analisam dependência para as AVD e funcionalidade em pessoas idosas ${ }^{(13,19)}$. Um estudo realizado com 156 idosos institucionalizados na Espanha, cuja amostra era predominantemente feminina, apesar de diferenças metodológicas na avaliação destas funções pela utilização da escala de Barthel, apontou para resultados semelhantes na avaliação das AVD, indicando dependência para a atividade de vestir-se e banhar$\mathrm{se}^{(20)}$.

Na avaliação da atividade banhar-se a dependência encontrada em nosso estudo com resultado estatisticamente significativo corrobora com outras pesquisas nas quais os achados revelam que o banho é a AVD menos realizada, tanto para os idosos que vivem em contextos de baixa e média vulnerabilidade social, como para os de alta e muito alta vulnerabilidade social, um indicativo de que esta atividade apresenta o menor percentual de independência(17,21).

Evidenciou-se no estudo associação significativa para as funções de banhar-se, vestir e ir ao banheiro com a necessidade de cuidado autorreferida, todavia, as demais AVD's: transferência, continência e alimentação, foram funções encontradas relativamente mais preservadas entre os indivíduos, concordando com outros estudos nos quais, dentre as atividades básicas da vida diária investigadas, os idosos apresentaram maior percentual de independência para comer, igualmente para a transferência e a continência ${ }^{(10,17,20)}$.

Nesse contexto, a avaliação da capacidade funcional torna-se essencial, pois detectada as limitações dependência/independência, a equipe de saúde pode reestruturar suas ações no sentido de prevenção, pois a perda da funcionalidade acarreta alterações na integridade da pessoa e aumenta a sobrecarga dos 
responsáveis pelo cuidado ${ }^{(22)}$. A literatura atesta que, tal quadro pode ser revertido e mutável mediante intervenção ${ }^{(19)}$.

Um dado do estudo que nos chamou a atenção é o fato de que enquanto $76,0 \%$ do total acusou dependência para alguma AVD, nas constatações referentes à necessidade de cuidado autorreferida, a atividade banhar-se foi significativamente mais preservada pelas religiosas, sugerindo que tal atitude pode estar relacionada ao pudor e constrangimento presentes entre os idosos, no tocante à exposição do corpo ao seu cuidador, mesmo que seja uma pessoa de sua intimidade ou profissional de saúde ${ }^{(7,23)}$ Vale salientar a severa formação à VRC que estas religiosas idosas foram expostas no passado, além da austeridade da vida conventual em seu cotidiano, vivenciado por décadas pelas mesmas. Se por um lado para o cuidador, a exposição do corpo do ser cuidado se reveste de certa naturalidade, até mesmo uma conotação de "banalização", para a religiosa que necessita de cuidado o sentido pode ser de sacralidade violada, a exposição corporal espelha a invasão da sua privacidade ${ }^{(23)}$.

\section{CONCLUSÕES}

Buscando atender o objetivo o estudo versou sobre a necessidade de cuidado e as condições de funcionalidade entre idosas religiosas as quais apresentaram níveis sociodemográficos elevados quando comparados aos resultados de estudos realizados com outros grupos de mulheres nessa faixa etária.

Constataram-se prevalências significantes nos relatos de necessidade de autocuidado relacionado ao desempenho das AVD banhar-se, vestir-se e ir ao

\section{REFERÊNCIAS}

1. Pedreira LC, Lopes RLM. Vivência do idoso dependente no domicílio: análise compreensiva a partir da historicidade heideggeriana. Rev. Eletr. Enf. Goiás. 2012;14(2):304-12.

2. Benítez-Silva $\mathrm{H}, \mathrm{Ni} \mathrm{H}$. Health status and health dynamics in an empirical model of expected longevity. J Health Econ.

2008;27(3):564-84.

3. Mesquita GV. Morbimortalidade em idosos por fratura proximal do fêmur. Texto Contexto Enferm. 2009;18(1):63-7.

4. Trigás-Ferrín M, Ferreira-González L, Meijide-Míguez $H$. Escalas de valoración funcional en el anciano. Galicia Clin. $2011 ; 72(1): 11-16$

5. Wolff JL, et al. Effects of Guided Care on Family Caregivers. The Gerontologist. 2009; 50(4):459-70.

6. Instituto Brasileiro de Geografia e Estatística. Tábuas

Completas de Mortalidade do Brasil. Rio de Janeiro, 2010.

Disponível em: http://www.ibge.gov.br. Acesso em: 17 nov. 2012.

7. Silva MJ, et al. Avaliação do grau de dependência nas atividades de vida diária em idosos da cidade de Fortaleza Ceará. Acta Paul Enferm. 2006;19(2):201-6.

8. Ben-Ezra M, Shmotkin D. Predictors of mortality in the old-old banheiro, variáveis do Index de Katz sendo que as demais: continência, transferência e alimentar-se apresentaram níveis de dependência não significativos, sugerindo uma possível ligação desta proteção com as diferentes oportunidades oferecidas dentro da vida religiosa consagrada, contexto a que o grupo é pertinente e que oportuniza aos seus, acesso a bens e serviços de modo diferenciado da sociedade em geral. As mesmas apresentam alto índice de longevidade, fator relevante quando se trata de independência para as AVD.

Recomendam-se avanços na abordagem do assunto visando aprofundar tanto fatores identificados como exercendo efeito deletério quanto a fatores que exercem efeito protetor da independência e preservação do autocuidado e funcionalidade para as AVD entre mulheres idosas religiosas que apresentem alto índice de longevidade.

Guardadas as limitações do presente estudo de impossibilidade de generalização de seus resultados, por se tratar de um contexto específico, pode-se, contudo, recomendar a proposição de futuras investigações congregando cenários similares, bem como a utilização de outras abordagens, as quais versem sobre questões subjetivas como forma a aprofundar as condições de saúde e a conjuntura que permeia a vida religiosa em seu cotidiano.

\section{AGRADECIMENTOS}

Ao Programa de Pós-Graduação em Envelhecimento Humano da Universidade de Passo Fundo pela concessão da bolsa nesses dois anos de Mestrado.

in Israel: the Cross-sectional and Longitudinal Aging Study. J. Am. Geriatr. Soc. 2006;54(6):906-11.

9. Pelegrin $A K$, et al. Idosos de uma Instituição de Longa Permanência de Ribeirão Preto: níveis de capacidade funcional. Arq. Ciênc. Saúde. 2008;15(4):182-88.

10. Ministério da Saúde. Envelhecimento e saúde da pessoa idosa. Brasília (Brasil): Ministério da Saúde; 2006. n.19.

11. Pontes-Barros JF, et al. Avaliação da capacidade funcional de idosos institucionalizados na cidade de Maceió - Al. Rev. Bras. Promoç. Saúde. 2010;23(2)168-74.

12. Araújo MOPH, Ceolim MF. Avaliação do grau de independência de idosos residentes em instituições de longa permanência. Rev. Esc. Enferm. 2007;41(3):378-85.

13. Del Duca GF, Silva MC, Hallal PC. Incapacidade funcional para atividades básicas e instrumentais da vida diária em idosos. Rev. Saúde Publica. 2009;43(5):796-05.

14. Fielder MM, Peres KG. Capacidade funcional e fatores associados em idosos do Sul do Brasil: um estudo de base populacional. Cad. Saúde Pública. 2008;24(2):409-15.

15. Parahyba MI, Veras R, Melzer D. Incapacidade funcional entre as mulheres idosas no Brasil. Rev. Saúde Pública.

$2005 ; 39(3): 383-90$. 
16. Oliveira $\mathrm{PH}$; Mattos IE. Prevalência e fatores associados à incapacidade funcional em idosos institucionalizados no Município de Cuiabá, Estado de Mato Grosso, Brasil, 2009-2010. Epidemiol. Serv. Saúde. 2012;21(3): 395-406.

17. Del Duca GF, et al. Incapacidade funcional em idosos institucionalizados. Rev. Bras. Ativ. Fis. Saúde. 2011;16(2):12024.

18. Oliveira DLC, Goretti LC, Pereira LSM. O desempenho de idosos institucionalizados com alterações cognitivas em atividades de vida diária e mobilidade: estudo piloto. Rev. Bras. Fisioter. 2006;20(1):91-6.

19. Oliveira EA, et al. Capacidade Funcional de Idosas de Instituição de Longa Permanência no Município de João Pessoa, Paraíba, Brasil: Estudo Piloto. R Bras Ci Saúde. 2012; 16(s2):83$8 .$.

20. Fuente Sanz MM, et al. Perfil del anciano institucionalizado en residencias privadas de la ciudad de Soria. Fisioterapia (Madr.). 2012; 34(6): 239-44

21. Santos AA, Pavarini SCI. Funcionalidade de idosos com alterações cognitivas em diferentes contextos de vulnerabilidade social. Acta Paul Enferm. 2011;24(4):520-26.

22. Nakatani AYK, Silva LB, Bachion MM, Nunes DP. Capacidade funcional em idosos na comunidade e propostas de intervenções pela equipe de saúde. Rev. Eletr. Enf. 2009;11(1):144-50.

23. Bettinelli LA, Pomatti DM, Brock J. Invasão da privacidade em pacientes de UTI: percepções de profissionais. Revista Bioéthicos. 2010;4(1):44-50.

Artigo recebido em 11/12/2012.

Aprovado para publicação em 27/02/2014.

Artigo publicado em 30/09/2014. 